International Journal of Pure and Applied Mathematics

Volume 103 No. 3 2015, 429-437

ISSN: 1311-8080 (printed version); ISSN: 1314-3395 (on-line version)

url: http://www.ijpam.eu

doi: http://dx.doi.org/10.12732/ijpam.v103i3.5

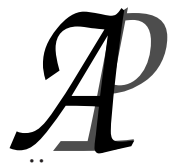

ijpam.eu

\title{
ON ONE-SIDED BASES OF A TERNARY SEMIGROUP
}

\author{
Boonyen Thongkam ${ }^{1}$, Thawhat Changphas ${ }^{2} \S$ \\ Department of Mathematics \\ Faculty of Science \\ Khon Kaen University \\ Khon Kaen, 40002, THAILAND
}

\begin{abstract}
In this paper, the notions of left bases and right bases of a ternary semigroup are introduced and the structure of a ternary semigroup containing right bases will be studied. For the structure of a ternary semigroup containing left bases can be considered similarly.
\end{abstract}

AMS Subject Classification: 20N15, 20N10

Key Words: ternary semigroup, left (right) ideal, left (right) base, right singular, maximality

\section{Preliminaries}

The notion of a ternary semigroup generalized the notion of a ternary group was defined as follows: a ternary semigroup (see, [7]) is a non-empty set $T$ together with a ternary operation, written as $(a, b, c) \rightarrow[a b c]$, satisfying the associative law, that is, for all $a, b, c, u, v \in T$,

$$
[[a b c] u v]=[a[b c u] v]=[a b[c u v]] .
$$

This notion has been widely studied (see [1], [2], [3], [4], [6], [7], [8], [9], [10], [11], [12]). If $A_{1}, A_{2}, A_{3}$ are non-empty subsets of a ternary semigroup $T$, the set product $\left[A_{1} A_{2} A_{3}\right]$ of $A_{1}, A_{2}, A_{3}$ is defined by:

$$
\left[A_{1} A_{2} A_{3}\right]=\left\{\left[a_{1} a_{2} a_{3}\right] \mid a_{1} \in A_{1}, a_{2} \in A_{2}, a_{3} \in A_{3}\right\} .
$$

Received: April 4, 2015

(C) 2015 Academic Publications, Ltd. url: www.acadpubl.eu

$\S_{\text {Correspondence author }}$ 
If $A_{1}=\{a\}$, we write $\left[A_{1} A_{2} A_{3}\right]$ as $\left[a A_{2} A_{3}\right]$, and similarly for $A_{2}=\{a\}$ or $A_{3}=\{a\}$. A non-empty subset $A$ of $T$ is called a ternary subsemigroup of $T$ if $[A A A] \subseteq A$. That is, if $\left[a_{1} a_{2} a_{3}\right] \in A$ for all $a_{1}, a_{2}, a_{3} \in A$.

The ideal theory of ternary semigroups was defined (see, [11], [7]) as follows: a non-empty subset $A$ of a ternary semigroup $T$ is called a left ideal (resp. right ideal) of $T$ if $[T T A] \subseteq A$ (resp. $[A T T] \subseteq A$ ). A left ideal $A$ if $T$ is said to be proper if $A \subset T$. The symbol $\subset$ stands for proper inclusion for sets.

It is known (see, [9], [11]) that the following hold for a non-empty subset $A$ of a ternary semigroup $T$ :

- $A_{\uparrow}=A \cup[T T A]$ is the left ideal generated by $A$ of $T$;

- $A_{\mathrm{r}}=A \cup[A T T]$ is the right ideal generated by $A$ of $T$. If $A=\{a\}$, we write $A_{\mathrm{l}}$ (resp. $A_{\mathrm{r}}$ ) as $(a)_{\mathrm{I}}$ (resp. $(a)_{\mathrm{r}}$ ).

We introduce the quasi-ordering on a ternary semigroup $T$ by: for any $a, b$ in $T$,

$$
a \leq_{\mathrm{I}} b \text { if and only if }(a)_{\mathrm{I}} \subseteq(b)_{\mid} .
$$

Tamura [13] introduced the notions of left bases and right bases of a semigroup. Fabrici [5] studied the structure of a semigroup containing one-sided bases. In this paper, we introduce the notions of one-sided bases, left bases and right bases, of a ternary semigroup and study the structure of a ternary semigroup containing right bases. For the structure of a ternary semigroup containing left bases can be considered dually.

\section{One-Sided Bases}

We define left and right bases of a ternary semigroup as follows.

Definition 1. A subset $A$ of a ternary semigroup $T$ is called a right base (resp. left base) of $T$ if it satisfies the following conditions:

(i) $A_{l}=T\left(\right.$ resp. $\left.A_{\mathrm{r}}=T\right)$;

(ii) there exists no a proper subset $B$ of $A$ such that $B_{\mid}=T\left(\right.$ resp. $\left.B_{\mathrm{r}}=T\right)$.

We now provide some examples.

Example 2. Let $T=\{-i, 0, i\}$. Then $T$ is a ternary semigroup under the multiplication over complex numbers (see, [3]). We have $\{i\}$ and $\{-i\}$ are both the left and right bases of $T$. 
Example 3. Let $T=\{0, a, b, c, 1\}$. Define the ternary operation on $T$ by, for all $a, b, c \in T,[a b c]=a *(b * c)$ where $*$ is the binary operation on $T$ defined by

\begin{tabular}{c|ccccc}
$*$ & 0 & $a$ & $b$ & $c$ & 1 \\
\hline 0 & 0 & 0 & 0 & 0 & 0 \\
$a$ & 0 & 0 & 0 & $a$ & $a$ \\
$b$ & 0 & 0 & $b$ & $b$ & $b$ \\
$c$ & 0 & 0 & $b$ & $c$ & $c$ \\
1 & 0 & $a$ & $b$ & $c$ & 1
\end{tabular}

Then $T$ is a ternary semigroup [2]. We have $\{1\}$ is a right and a left base of $T$.

Example 4. Let $T$ be a non-empty set such that $|T| \geq 2$. Then $T$ is a ternary semigroup under the ternary operation which is defined by $[x y z]=x$ for all $x, y, z \in T$. This is called the left zero ternary semigroup [10]. Then, for all $a \in T,\{a\}$ is a right base of $T$.

Example 5. Let $T$ be a non-empty set such that $|T|>3$. Choose an element $0 \in T$ and define the ternary operation on $T$ by, for any $x, y, z \in T$,

$$
[x y z]= \begin{cases}x & \text { if } x=y=z \\ 0 & \text { otherwise }\end{cases}
$$

Then $T$ is a ternary semigroup [10]. We have $T \backslash\{0\}$ is both the right and the left base of $T$.

Example 6. Let $T=\{0,1,2,3,4,5\}$. Define the ternary operation on $T$ by, for all $a, b, c \in T,[a b c]=(a * b) * c$ where $*$ is the binary operation on $T$ defined by

\begin{tabular}{c|cccccc}
$*$ & 0 & 1 & 2 & 3 & 4 & 5 \\
\hline 0 & 0 & 0 & 0 & 0 & 0 & 0 \\
1 & 0 & 1 & 1 & 1 & 1 & 1 \\
2 & 0 & 1 & 2 & 3 & 1 & 1 \\
3 & 0 & 1 & 1 & 1 & 2 & 3 \\
4 & 0 & 1 & 4 & 5 & 1 & 1 \\
5 & 0 & 1 & 1 & 1 & 4 & 5
\end{tabular}

Then $T$ is a ternary semigroup [10]. We have $\{2,3\},\{2,5\},\{3,4\},\{4,5\}$ are the right bases of $T$. And, $\{2,4\},\{2,5\},\{3,4\},\{3,5\}$ are the left bases of $T$.

We now prove some elementary results. 
Lemma 7. Let $A$ be a right base of a ternary semigroup $T$. If $a, b \in A$ and $a \in[T T b]$, then $a=b$.

Proof. Let $a, b \in A$ be such that $a \in[T T b]$. Suppose that $a \neq b$. Let $B=A \backslash\{a\}$. Then $B \subset A$. Let $x \in A$. If $x \notin B$, then

$$
x=a \in[T T b] \subset[T T B] \cup B .
$$

Thus

$$
A \subseteq[T T B] \cup B \subseteq B_{\text {। }}
$$

Hence $T=A_{\mid} \subseteq B_{\mid}$. This is a contradiction. Thus $a=b$.

Lemma 8. A non-empty subset $A$ of a ternary semigroup $T$ is a right base of $T$ if and only if $A$ satisfies the following conditions:

(1) for any $x \in T$ there exists $a \in A$ such that $x \leq_{1} a$;

(2) for any $a, b \in A$, if $a \neq b$, then neither $a \leq_{1} b$ nor $b \leq_{1} a$.

Proof. Assume that $A$ is a right base of $T$. Let $x \in T$. Then $x \in A_{\mid}=$ [TTA] $\cup A$, that is, $x \in A$ or $x \in[T T A]$. If $x \in A$, then there exists $a=x \in A$ such that $x \leq_{\mathrm{I}} a$. If $x \in[T T A]$, then $x=[s t a] \in(a)$ । for some $s, t \in T, a \in A$. Thus $(x)_{\mid} \subseteq(a)_{\mid}$. This shows that (1) holds. Let $a, b \in A$ be such that $a \neq b$. Suppose that $a \leq_{\mid} b$. Then $(a)_{\mid} \subseteq(b)_{\mid}$. Since $a \neq b$, we have $a \in(b)_{\mid} \backslash\{b\}$. By Lemma 7, $a=b$. This is a contradiction. Similarly, $b \leq_{1} a$ implies $a=b$. This is a contradiction. Therefore, (2) holds.

Conversely, assume that the conditions (1) and (2) hold. It follows from (1) that $T=A_{\uparrow}$. Suppose that $T=B_{\text {। }}$ for some a proper subset $B$ of $A$. Let $a \in A \backslash B$. We have $a \in B_{\mid}$. By (1), there exists $b \in B$ such that $a \leq_{1} b$. This contradicts to (2). Hence $A$ is a right base of $T$.

\section{Main Results}

A ternary semigroup $T$ is said to be right singular if, for any $x, y, z \in T$, $[x y z]=z$. An element $a$ of $T$ is called an selfpotent if $[a a a]=a$.

In general, a right base of a ternary semigroup need not be a ternary subsemigroup. The following theorem characterizes when a right base of a ternary semigroup is a ternary subsemigroup.

Theorem 9. Let $A$ be a right base of a ternary semigroup T. Then $A$ is a ternary subsemigroup of $T$ if and only if $A$ is right singular. 
Proof. Assume that $A$ is a ternary subsemigroup of $T$. Let $a, b, c \in A$. Thus $[a b c] \in A$, and so $[a b c]=d$ for some $d \in A$. Then $d \in[T T c]$. By Lemma 7 , $d=c$. Therefore, $A$ is right singular.

Conversely, if $A$ is right singular, then, for $a, b, c \in A,[a b c]=c \in A$. Hence $A$ is a ternary subsemigroup of $T$.

By Theorem 9, we have the following.

Corollary 10. If a right base $A$ of a ternary semigroup $T$ is a ternary subsemigroup of $T$, then $T$ contains at least one selfpotent.

The following theorem shows that for any two right bases of a ternary semigroup have the same cardinality.

Theorem 11. Any two right bases of a ternary semigroup $T$ have the same cardinality.

Proof. Let $A$ and $B$ be right bases of a ternary semigroup $T$. Let $a \in A$. Since $B$ is a right base of $T$, we have $a \leq_{1} b$ for some $b \in B$. For $a \in A$, we choose and fix $b \in B$ such that $a \leq_{1} b$ and define a mapping

$$
f: A \rightarrow B \text { by } f(a)=b \text { for all } a \in A \text {. }
$$

If $a_{1}, a_{2} \in A$ such that $f\left(a_{1}\right)=f\left(a_{2}\right)=b$, then $a_{1} \leq_{1} b$ and $a_{2} \leq_{1} b$. Since $A$ is a right base of $T$, we have $b \leq_{1} a^{\prime}$ for some $a^{\prime}$ in $A$. Thus $a_{1} \leq_{1} a^{\prime}, a_{2} \leq_{1} a^{\prime}$, and $a_{1}, a_{2}, a^{\prime} \in A$. Thus $a_{1}=a^{\prime}=a_{2}$ by Lemma 8. Hence $f$ is one to one.

Now, let $b \in B$. Then there exists $a \in A$ such that $b \leq_{1} a$. Similarly, there exists $b^{\prime} \in B$ such that $a \leq_{1} b^{\prime}$. Then $b \leq_{1} b^{\prime}$. By Lemma 8 , we have $b=b^{\prime}$. Thus $a \leq_{1} b^{\prime}=b$. Let $f(a)=c$. Then $a \leq_{1} c$ and $a \leq_{1} b$. Since $c, b \in T$ and $A$ is a right base of $T$, there exist $a^{\prime}, a^{\prime \prime} \in A$ such that $c \leq_{1} a^{\prime}$ and $b \leq_{1} a^{\prime \prime}$. Then $a \leq_{1} a^{\prime}$ and $a \leq_{1} a^{\prime \prime}$. By Lemma $8, a=a^{\prime}=a^{\prime \prime}$. Then $b \leq_{1} a^{\prime \prime}=a \leq_{1} c$, and so $b=c$ by Lemma 8 . Hence $f$ is onto.

Lemma 12. Let $A$ be a right base of a ternary semigroup $T$. Let $a \in A$. If $(a)_{\mid}=(b)_{\mid}$for some $b \in T$ and $b \neq a$, then $b$ is an element of a right base of $T$ which is distinct from $A$.

Proof. Let $B=(A \backslash\{a\}) \cup\{b\}$. It is clear that $B \subset A$. To show that $B$ is a right base of $T$, it suffices to show that $B$ satisfies the conditions (1) and (2) of Lemma 8. Let $x \in T$. Since $A$ is a right base of $T$, there exists $c \in A$ such that $x \leq_{\mid} c$. If $c \neq a$, then $c \in B$. If $c=a$, then $(c)_{\mid}=(a)_{\mid}$. Thus $(c)_{\mid}=(b)_{\mid}$, and so

$$
(x)_{\mathrm{I}} \subseteq(c)_{\mathrm{I}}=(b)_{\mathrm{I}} .
$$


It follows that $x \leq_{1} b$ and $b \in B$. It means that $B$ satisfies the condition (1) of Lemma 8. Now, let $b_{1}, b_{2} \in B$ be such that $b_{1} \neq b_{2}$. If $b_{1}, b_{2}$ are distinct from $b$, then $b_{1}, b_{2} \in A$. Since $A$ is a right base of $T$, so neither $b_{1} \leq_{1} b_{2}$ nor $b_{2} \leq_{1} b_{1}$. Let $b_{1}=b$. If $b_{1} \leq_{1} b_{2}$, then $a \leq_{1} b_{2}$ where $a, b_{2} \in A$. This is impossible. If $b_{2} \leq_{\mathrm{l}} b_{1}$, then $\left(b_{2}\right)_{\mathrm{I}} \subseteq\left(b_{1}\right)_{\mathrm{l}}=(a)_{\mid}$. Thus $b_{2} \leq_{\mathrm{|}} a$ where $a, b_{2} \in A$. This is impossible. It means that $B$ satisfies the condition (2) of Lemma 8. Therefore, $B$ is a right base of $T$.

This is a consequence of the lemma above.

Corollary 13. Let $A$ be a right base of a ternary semigroup $T$. Let $a \in A$. If $(a)_{\mid}=(b)_{\mid}$for some $b \in T$ and $b \neq a$, then $T$ contains at least two right bases.

Theorem 14. Let $\mathcal{A}$ be the union of all right bases of a ternary semigroup $T$. If $L=T \backslash \mathcal{A}$ is non-empty, then $L$ is a left ideal of $T$.

Proof. Let $x, y \in T$ and $a \in L=T \backslash \mathcal{A}$. Suppose that $[x y a] \notin L$. Then there exists $b \in \mathcal{A}$ such that $b=[x y a] \subseteq[T T a]$. That is, $(b)_{\mid} \subseteq(a)_{\mid}$. Then $b \leq_{1} a$. Since $b \in \mathcal{A}$, so $b \in B$ for some a right base $B$ of $T$. Since $B$ is a right base, there exists $b_{1} \in B$ such that $a \leq_{1} b_{1}$. Then $b \leq_{1} a \leq_{1} b_{1}$, and thus $b \leq_{1} b_{1}$. This contradicts to the condition (2) of Lemma 8. Hence $[x y a] \in L$.

A proper left ideal $M$ of a ternary semigroup $T$ is said to be maximal if there is no a proper left ideal $A$ of $T$ such that $M \subset A$.

Theorem 15. Let $\mathcal{A}$ be the union of all right bases of a ternary semigroup $T$ such that $\mathcal{A} \neq \emptyset$. Then $T \backslash \mathcal{A}$ is a maximal proper left ideal of $T$ if and only if $\mathcal{A} \neq T$ and $\mathcal{A} \subseteq(a)$, for all $a \in \mathcal{A}$.

Proof. Let $L=T \backslash \mathcal{A}$ be a maximal proper left ideal of a ternary semigroup $T$. Then $\mathcal{A} \neq T$. Let $a \in \mathcal{A}$. Suppose that $\mathcal{A} \nsubseteq(a)_{\text {| }}$. Since $\mathcal{A} \nsubseteq(a)_{\text {I }}$, there exists $x \in \mathcal{A}$ such that $x \notin(a)_{\mid}$. Thus $x \notin T \backslash \mathcal{A}$ and $x \notin(a)_{\mid}$. This implies $(T \backslash \mathcal{A}) \cup(a)_{\mid} \neq T$. Then $(T \backslash \mathcal{A}) \cup(a)_{\mid} \neq T$ is a proper left ideal of $T$ such that $(T \backslash \mathcal{A}) \subset(T \backslash \mathcal{A}) \cup(a)_{\mid}$. This contradicts to the maximality of $T \backslash \mathcal{A}$. Hence $\mathcal{A} \subseteq(a)_{1}$

Conversely, let $\mathcal{A} \subseteq(a)$, for all $a \in \mathcal{A}$, and $\mathcal{A} \neq T$. We have to prove that $T \backslash \mathcal{A}$ is a maximal proper left ideal of $T$. By Theorem $14, T \backslash \mathcal{A}$ is a proper left ideal of $T$. Let $L^{\prime}$ be a left ideal of $T$ such that $T \backslash \mathcal{A} \subset L^{\prime}$. Then $L^{\prime} \cap \mathcal{A} \neq \emptyset$. Let $a \in L^{\prime} \cap \mathcal{A}$. Then $a \in L^{\prime}$, and it follows that $(a)_{\mid} \subseteq L^{\prime}$. Since $\mathcal{A} \subseteq(a)_{\text {। }}$, $\mathcal{A} \subseteq L^{\prime}$. Consequently, $\mathcal{A} \subseteq L^{\prime}, T \backslash \mathcal{A} \subset L^{\prime}$. Therefore $T=L^{\prime}$. 
Theorem 16. Let $\mathcal{A}$ be the union of all right base of a ternary semigroup $T$ such that $\emptyset \neq \mathcal{A} \subset T$. Let $M^{*}$ be a proper left ideal of $T$ containing every proper left ideal of $T$. The following statements are equivalent:

(1) $T \backslash \mathcal{A}$ is a maximal left ideal of $T$;

(2) $\mathcal{A} \subseteq(a)$ । for every $a \in \mathcal{A}$;

(3) $T \backslash \mathcal{A}=M^{*}$;

(4) every right base of $T$ is a singleton set.

Proof. (1) $\Leftrightarrow(2)$. This follows from Theorem 15 .

(3) $\Leftrightarrow(4)$. Assume that $T \backslash \mathcal{A}=M^{*}$. Then $T \backslash \mathcal{A}$ is a maximal left ideal of $T$. Let $a \in \mathcal{A}$. By Theorem 15, we have $\mathcal{A} \subseteq(a)_{\mid}$. Then $T=\mathcal{A}_{\mid} \subseteq(a)_{\mid}$. This implies $T=(a)_{\mid}$. Hence $\{a\}$ is a right base of $T$. Let $B$ be a right base of $T$, and let $a, b \in B$. Then $B \subseteq \mathcal{A}$, that is, $a, b \in \mathcal{A}$. Hence $b \in T=(a)_{\text {I. }}$ By Lemma $7, a=b$ (i.e., $B$ is a singleton set).

Conversely, assume that every right base of $T$ is a singleton set. Then

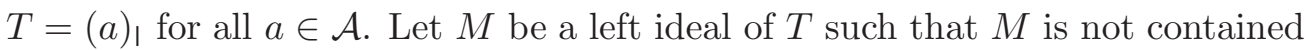
in $T \backslash \mathcal{A}$. Then there exists $x \in \mathcal{A} \cap M$. Since $x \in M$, we have $T=(x)_{\mid} \subseteq M$, and so $T=M$.

$(1) \Leftrightarrow(3)$. Assume that $T \backslash \mathcal{A}$ is a maximal left ideal of $T$. Let $M$ be a left ideal of $T$ such that $M$ is not contained in $T \backslash \mathcal{A}$. Hence, there exists $x \in M \cap \mathcal{A}$. Then $\mathcal{A} \subseteq(x)_{1} \subseteq M$. Thus $M=\mathcal{A} \cup X$ for some $X \subseteq T \backslash \mathcal{A}$. Let $y \in T$. Then there exists $c \in \mathcal{A}$ such that $y \leq c$; hence $y \in(y)_{\perp} \subseteq(c)_{I} \subseteq M$. Thus $M=T$. Therefore, $T \backslash \mathcal{A}=M^{*}$.

The converse statement is obvious.

Theorem 17. Let $\mathcal{A}$ be the union of all right bases of a ternary semigroup $T$. If $\emptyset \neq \mathcal{A} \neq T$ and $T \backslash \mathcal{A}$ is a maximal left ideal of $T$, then every right base $A$ of $T$ from neither $[T T A]=T$ (i.e., $A_{\uparrow}=[T T A]$ ) or there is unique a right base $A$ of $T$ such that $A \subseteq T \backslash[T T A]$.

Proof. Assume that $T \backslash \mathcal{A}$ is a maximal left ideal of $T$ and $A$ is a right base of $T$. By Theorem 16, $A=\{a\}$ for some $a \in T$ and $\mathcal{A} \subseteq(x)$ । for all $x \in \mathcal{A}$. If there exist $x, y \in \mathcal{A}$ such that $x \in[T T x]$ and $y \notin[T T y]$, then

$$
T \backslash \mathcal{A} \subseteq[T T y] \subset T
$$

where $T \backslash \mathcal{A} \neq[T T y]$, because $x \in[T T y]$. Hence $T \backslash \mathcal{A} \neq[T T y]$. This is contradicts to the maximality of $T \backslash \mathcal{A}$. Hence, there are two cases to consider:

Case 1: $x \in[T T x]$ for all $x \in \mathcal{A}$. We have $[T T A]=T$. That is, $A_{\mid}=[T T A]$. 
Case 2: $x \notin[T T x]$ for all $x \in \mathcal{A}$. We have $A \subseteq T \backslash[T T A]$. Suppose that $T$ contains at least two right bases, $A_{1}=\left\{a_{1}\right\}, A_{2}=\left\{a_{2}\right\}$, such that $a_{1} \notin\left[T T a_{1}\right], a_{2} \notin\left[T T a_{2}\right]$ and $a_{1}, a_{2} \in \mathcal{A}$. Thus

$$
T \backslash \mathcal{A} \subseteq T \backslash\left\{a_{1}\right\}=\left[T T a_{1}\right] .
$$

Since $a_{2} \in\left[T T a_{1}\right], T \backslash \mathcal{A} \neq\left[T T a_{1}\right]$. This contradicts to the maximality of $T \backslash \mathcal{A}$. Hence, there is unique a right base $A$ of $T$ such that $A \subseteq T \backslash[T T A]$.

\section{References}

[1] M. Amyari and M. S. Moslehian, Approximate homomorphisms of ternary semigroups, Letters Math. Physics, 77(2006), 1-9.

[2] S. Bashir and M. Shabir, Pure ideals in ternary semigroups, Quasigroups and Related Systems, 17(2009), 149-160.

[3] V. N. Dixit and S. Dewan, A note on quasi and bi-ideals in ternary semigroups, Int. J. Math. Math. Sci., 18(1995), 501-508.

[4] V. N. Dixit and S. Dewan, Minimal quasi-ideals in ternary semigroups, Indian J. Pure Appl. Math., 28(1997), 255-632.

[5] I. Fabrici, One-sided bases of semigroups, Matematický časopis, 22 (1972), 286-290.

[6] R. Kerner, Ternary algebraic structures and their applications in Physics, Univ, P\&M, Curie Preprint, Paris (2000), Arxiv math-ph/0011023.

[7] J. Los, On the extending of models I, Fundamenta Mathematicae, 42(1955), 38-54.

[8] M. L. Santiago, Some contributions to the study of ternary semigroups and semiheaps, Ph.D. Thesis, 1983, University of Madras.

[9] M. L. Santiago, S. Sri Bala, Ternary semigroups, Semigroup Forum, 81(2010), 380-388.

[10] M. Shabir and M. Bano, Prime bi-ideals in ternary semigroups, Quasigroups and Related Systems, 16(2008), 239-256.

[11] F. M. Sioson, Ideal theory in ternary semigroups, Math. Japonica, 10 (1965), 63-84. 
[12] M. Shabir and S. Bashir, Prime ideals in ternary semigroups, Asian European J. Math., 2(2009), 139-152.

[13] T. Tamura, One-sided bases and translations of a semigroup. Math. Japan., 3(1955), 137-141. 
